\title{
Theme Park Mobility in Disaster Scenarios
}

\author{
Gürkan Solmaz and Damla Turgut \\ Department of Electrical Engineering and Computer Science \\ University of Central Florida \\ Email: \{gsolmaz,turgut\}@eecs.ucf.edu
}

\begin{abstract}
In this paper, we propose a scenario-specific human mobility model (TP-D) in theme parks. We focus on the disaster scenarios which have significant differences compared to the ordinary mobility behavior of the theme park visitors. The main goal of the theme park operation in disaster scenarios is the evacuation of the visitors from the disaster areas.

We first model theme park as a combination of roads, obstacles, lands, and disaster events. We use real theme park maps for generating the theme park models. We incorporate the macro and micro mobility behaviors of the visitors in theme park models. We use the social force concept to model the impact of social interactions on the micro mobility of the visitors. Macro mobility decisions are based on the local knowledge of the visitors, the waypoints, and the disaster events. We analyze and compare the results of the simulation of our model with simulations of currently used models and real-world GPS traces of visitor movement.
\end{abstract}

\section{INTRODUCTION}

Realistic modeling and accurate simulation of the mobility in theme parks allows us to evaluate performance of the networks for event coverage [1], the opportunistic communication [2], and evacuation of the visitors from disaster areas.

Human mobility is based on the combinations of many factors including deterministic and non-deterministic decisions which depend on the scenario. Most commonly used simple mobility models such as random waypoint (RWP) model or generic human mobility models are not suitable to represent the human mobility behavior in many different application scenarios. There is a certain need for scenario-specific modeling for realistic simulations of human mobility. Therefore, we modeled visitor movements in theme parks in [3] to represent daily routine mobility of theme park visitors without any consideration of the disaster scenarios. However, in our model [3] and the other currently used theme park mobility models, the movement decisions of the visitors are based on visiting the attractions and exploring the park. Considering a disaster scenario, the movement decisions should be based on the security of visitors. The main goal of the theme park operation include finding easy ways to secure places and fast evacuation of the visitors from the disaster areas.

We consider a wide range of disaster scenarios which may happen in theme parks. These scenarios include natural disasters such as tornado, fire, thunderstorm, hurricane, and earthquake. Moreover, terrorist attacks are man-made disasters which threaten human life especially in crowded places. Therefore, security of the theme parks has vital importance. The studies on disaster recovery and opportunistic communication networks have become major research interests due to their prospective contributions on the disaster management strategies. For instance, as an effect of a disaster, communication infrastructures which are pre-deployed in the disaster area may become unoperational. For this reason, communication systems independent from the infrastructures are taken into account in many disaster management studies. Crowd management and evacuation of people from disaster areas are other major challenges which have theoretical and practical interests from the research community. Modeling disaster mobility in theme parks is useful for finding novel methods to solve the evacuation problem in theme parks. In addition, these methods may become the base-case for the evacuation problem of more complicated scenarios such as evacuation of people from buildings and evacuation from big cities.

Let us now consider the possible uses of the proposed mobility model in real life applications. The mobility model can be used to evaluate performance of the wireless ad hoc networks. Opportunistic message forwarding through the smart-phones to increase awareness of the people in a disaster scenario is possible with the use of these networks. The performance of the networks highly rely on the mobility of the people and can be evaluated before creating the real applications. Moreover, various crowd management and evacuation strategies for theme parks can be tested using the simulations of this model. The simulations of this model can be essentially used for determining the positions of exit gates and finding strategic points for crowd management to place security precautions.

In this paper, we present the mobility of visitors in theme parks for disaster scenarios. We model theme park as a combination of roads, obstacles, lands, and red-zones using real theme park maps. To model the visitor movements, we consider the macro and micro mobility decision problems separately. We use the social force model [4] to represent the dynamics of the human motion by the social interactions. Finally, we analyze the results of our model and compare it with the currently used mobility models and the GPS traces of theme park visitors.

\section{Mobility Model}

\section{A. Modeling the theme park}

Let us briefly describe the fundamental characteristics of theme parks by looking from the mobility modeling perspective. Theme parks consist of attractions which are entertainment places including rides, restaurants, and places for other activities. Attractions consist of man-made structures (i.e. buildings) and they are connected to each other by roads. The roads also connect the entrance and exit points of the theme park with the attractions. They are usually used by only pedestrians, specific for theme park environment. Each road has a width which determines the capacity of the road for pedestrian flows. For instance, if a road is narrow and there are many people, the density of the people becomes large and as a result people cannot move fast enough along the road. 


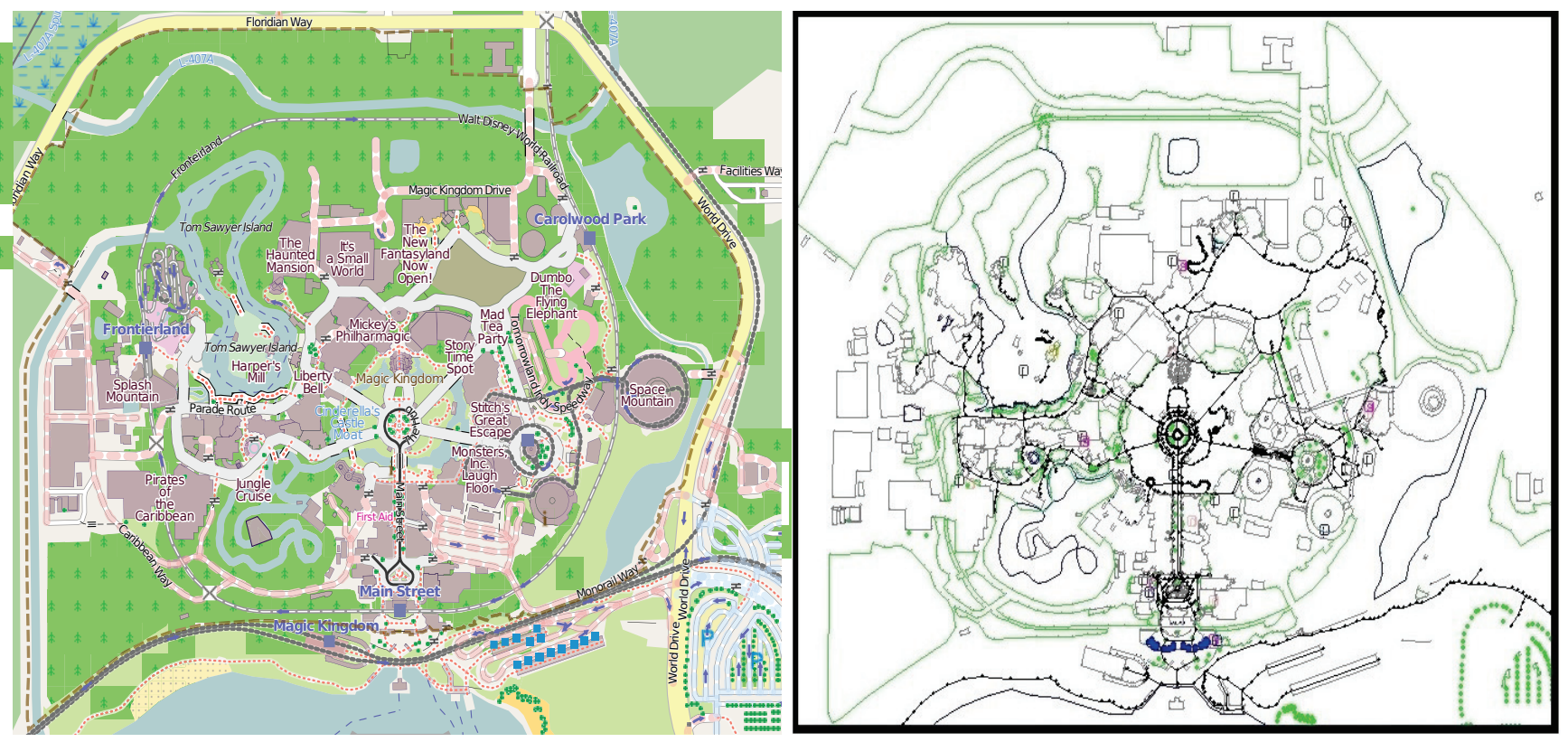

Fig. 1. The maps of the Magic Kingdom park. Left: the map extracted from (OSM), right: the processed map with 1300 waypoints.

We first start describing the theme park model for disaster scenarios in detail. We model the theme park as the combination of roads, obstacles, lands, and disaster events. Each road contains a set of waypoints, which are the movement points for the theme park visitors. In this case, length of a road is equal to the sum of the distances between each pair of its consecutive waypoints. The roads show the possible ways to reach the target locations in the map. The gates are considered as the target locations and they are placed close to the borders of the park. The gates connect the theme park with the outside world and facilities such as transportation vehicles (i.e. ambulances, fire engines).

As mentioned, attractions contain man-made buildings and other structures. In the ordinary times, the main goal of the visitors is to visit the attractions. For a disaster case, when the visitors should be evacuated from the disaster area as quickly as possible, we consider these buildings as the obstacles which prevent the free movement of the visitors. Furthermore, we model the other man-made structures in the park including fences, walls as the obstacles. There are also natural obstacles such as lakes, trees, forest, river, and so on. We do not consider the evacuation problem from the buildings and assume that each visitor is outside.

The areas which neither include the obstacles nor the roads are classified as the lands. The lands can be used by pedestrians but they are not preferred unless there are unexpected conditions on the available roads. For instance, when a road is unavailable due to an impact of the disaster event, the lands might be used instead. In some cases, lands provide shortcuts between the waypoints. Disaster areas are classified as the redzones and they are the circular areas reflecting the effects of the disaster. In a real scenario, one can think the red-zones as the events which damage roads or bridges, caused by an earth-quake, a hurricane, a fire, a terrorist attack and so on. The red-zones have radius values which specify the damaged areas and active times. If a red-zone is in its active time and it effects an area including some portions of a road, the road is assumed to be unavailable at that particular time.

The model of the theme park can be created synthetically or using real maps. We use OpenStreetMap (OSM) [5] to extract the real theme park maps and parse the OSM data to generate the roads, the obstacles, the lands, and the gates. We collect the waypoints using the OSM data and connect the consecutive waypoints to create the roads. We assign width values to the roads according to their OSM types (footway, path, and pedestrian way). Fig. 1 displays an example of the real map of the Magic Kingdom park from the Disney World in Orlando (left-side), and the processed version of the map including the waypoints, the roads, the gates, and the obstacles (right-side). In this figure, the small black dots represent the waypoints, while the black lines connecting the waypoints are the roads and the closed polygons are the obstacles. The model also include red-zones which are added to the model according to their active times; however, they are not included in this initial processing of the map. The two gates can be seen at the bottom part of the figure as the two thick blue lines close to each other. The generation of the theme park models are done computationally, but it is possible to create a non-existing theme park in design stage manually and create the theme park model in the same fashion.

\section{B. Modeling mobility of the visitors}

We describe the mobility behavior of the visitors as follows. The visitors have the local knowledge of their environments and the knowledge of the position of the gate which they are entered through. The local knowledge of the visitors is determined by the visibility parameter which shows the visible distance for each visitor and the obstacles which may be located along the way. The visibility parameter represents the radius of the circular visible area. The visitors are not assumed to communicate with each other and there is no broadcasting system for raising the awareness. 


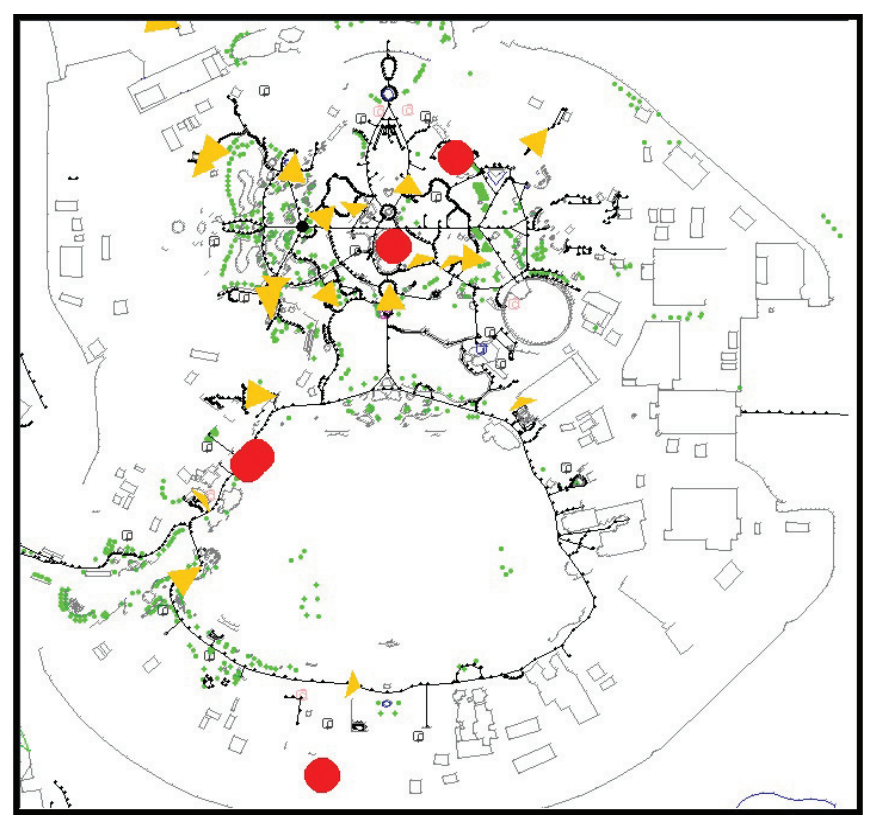

Fig. 2. Epcot theme park model including 20 visitors, 2300 waypoints, and 5 red-zones.

Initially, the visitors are randomly distributed to one of the waypoints in the theme park model. Each visitor selects an exit gate among the available exit gates in the park and mark its position as the target point. A visitor is assumed to be evacuated after reaching one of the exit gates. The visitor tries to reach the target point by moving among the waypoints. Whenever the visitor reaches a waypoint, marks it as visited. The next destination point is selected among all the visible waypoints. The visited waypoints, the waypoints positioned in a red-zone or the waypoints which are not in the visible area of the visitor are not taken into consideration as the candidates for the next destination point. The visitor selects a new waypoint according to its distance and direction from the current position of the visitor since the visitor tries to select the destination point closer to the target. The selection of the waypoints is constrained by the the visitors knowledge about the world, obstacles, and possible active red-zones along the way. If a visitor cannot find any waypoint as a candidate for the next destination, the new destination is selected by exploration with a random direction. The random exploration distance is a parameter which bounds the flexibility of the movement of the visitors in cases of the unexpected disaster events. Another parameter which effects the flexibility the most is the visibility parameter. The visibility may differ according to the type and the impact of the disasters. We classify all the above steps of a visitor considering the global movement starting from the initial point to the target point as macro-mobility behavior of the visitors.

The speeds of the visitors differ from one to another. Basically, each visitor has a maximum speed which depends on physical attributes of the individual such as age, gender, and weight. The speed value for each individual is a random value between a global minimum and global maximum speed of the visitors. The speed of a visitor varies from its minimum, which is 0 to maximum speed. On the other hand, the global minimum value determines the minimum value for the maximum speed value of all visitors. The maximum speed is the speed when the visitor is completely free to walk without disturbance or the obstacles. In the disaster scenario, the actual speed of a visitor is less than the maximum speed most of the times due to the effects of the social interactions which are explained below.

Fig. 2 illustrates the complete theme park model generated using the map of the Epcot park in Disney World. In this figure, the visitors and the red-zones are included in the model. 20 visitors moving along the roads are represented by the yellow triangles. The shape of the triangles illustrate the directions and velocities of each of the visitors. 5 red-zones are represented by the big red circles. The two red-zones having an intersection area appeared as an enlargered red-zone, located in the middle of the figure.

We consider micro-mobility as the mobility of a visitor between the two consecutive waypoints separately from the macro-mobility model and the theme park model. We use the social force model (SFM) [4] which is used by the simulators such as SimWalk and VisSim for the micro-mobility. According to the social force concept, behavioral changes in the human motion can be explained and is actually caused by the combination of the social interactions. Using the SFM, we model the social forces on the visitors according to their social interactions with the environment. By this model, the visitors adapt their speed and direction of the movement from a waypoint to another. In SFM, the sum of the social forces is given by

$$
f_{\alpha}(t)=\frac{1}{\tau_{\alpha}}\left(v_{\alpha}^{0} e_{\alpha}^{0}-v_{\alpha}\right)+\sum_{\beta(\neq \alpha)} f_{\alpha \beta}(t)+\sum_{i} f_{\alpha i}(t),
$$

for a visitor $\alpha$ where $\tau_{\alpha}$ denotes the relaxation time, $v_{\alpha}^{0} e_{\alpha}^{0}$ is the desired velocity, and the sums correspond to the social forces by the other visitors $(\beta)$ and the obstacles $(i)$ respectively. The acceleration is then given by $f_{\alpha}(t)$ and the individual fluctuations. Assuming $f_{\alpha \beta}(t)=f\left(d_{\alpha \beta}(t)\right)$, circular specification is given by

$$
f\left(d_{\alpha \beta}\right)=A_{\alpha} e^{-d_{\alpha \beta} / B_{\alpha}} \frac{d_{\alpha \beta}}{\left\|d_{\alpha \beta}\right\|},
$$

where $A_{\alpha}, B_{\alpha}$ denote the interaction strength and the interaction range respectively.

For the elliptical specification of the model, the circular specification formula is expressed as a gradient of an exponential decaying potential $V_{\alpha \beta}$, where elliptical interaction force via the potential is $V_{\alpha \beta}\left(b_{\alpha \beta}\right)=A B e^{-b_{\alpha \beta} / B}$. In this equation, $b_{\alpha \beta}$ is the semi-minor axis of the elliptical equipotential lines and given by

$$
2 b_{\alpha \beta}=\sqrt{\left(\left\|d_{\alpha \beta}\right\|+\left\|d_{\alpha \beta}-y_{\alpha \beta}\right\|\right)^{2}-\left\|y_{\alpha \beta}\right\|^{2}},
$$

where $y_{\alpha \beta}=\left(v_{\beta}-v_{\alpha}\right) \Delta t$ and $\Delta t=0.5 s$.

$$
f_{\alpha \beta}=-\nabla_{d_{\alpha \beta}} V_{\alpha \beta}\left(b_{\alpha \beta}\right)=-\frac{d V_{\alpha \beta}\left(b_{\alpha \beta}\right)}{d b_{\alpha \beta}} \nabla_{d_{\alpha \beta}} b_{\alpha \beta}\left(d_{\alpha \beta}\right)
$$

Equation 4 gives the repulsive force and $\nabla_{d_{\alpha \beta}}$ denotes the gradient with respect to distance between $\alpha$ and $\beta$. Using chain rule, this leads to 


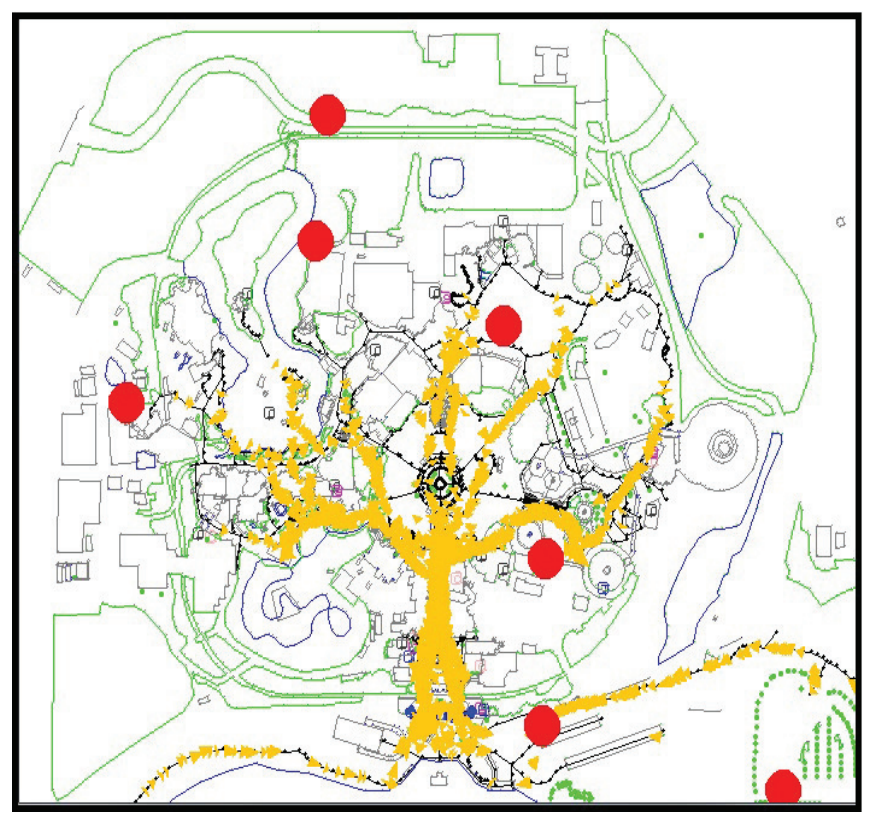

Fig. 3. The simulation of 2000 visitors and the impact of red zones in Magic Kingdom.

$$
\begin{array}{r}
f_{\alpha \beta\left(d_{\alpha \beta}\right)}=A_{\alpha} e^{-b_{\alpha \beta} / B} \cdot \frac{\left\|d_{\alpha \beta}\right\|+\left\|d_{\alpha \beta}-y_{\alpha \beta}\right\|}{2 b_{\alpha \beta}} \\
\cdot \frac{1}{2}\left(\frac{d_{\alpha \beta}}{\left\|d_{\alpha \beta}\right\|}+\frac{d_{\alpha \beta}-y_{\alpha \beta}}{\left\|d_{\alpha \beta}-y_{\alpha \beta}\right\|}\right) .
\end{array}
$$

Considering the angular dependence between two encountered visitors, with an angle of $\varphi_{\alpha \beta}$, the angular-dependent pre-factor $w\left(\varphi_{\alpha \beta}\right)$ is given by the below equations

$$
\begin{gathered}
\cos \left(\varphi_{\alpha \beta}\right)=\frac{v_{\alpha}}{\left\|v_{\alpha}\right\|} \cdot \frac{-d_{\alpha \beta}}{\left\|d_{\alpha \beta}\right\|} \\
w\left(\varphi_{\alpha \beta}(t)\right)=\left(\left(1-\lambda_{\alpha}\right) \frac{1+\cos \left(\varphi_{\alpha \beta}\right)}{2}+\lambda_{\alpha}\right),
\end{gathered}
$$

where the parameter $\lambda_{\alpha}$ with $0 \leq \lambda_{\alpha} \leq 1$ is found by evolutionary optimization as $\lambda_{\alpha} \approx 0.1$. The fitness of the social force model increases with the addition of the angular dependence formulation to the model.

As a consequence of this model, the time it takes for the visitor to move to a destination point differs. The main effect of this model in the theme park scenario is that the usage of the same roads by the visitors causes an increase in the social interactions. This increase slows down the flow of the visitors along the roads. Since the theme parks are crowded areas with roads in which only pedestrian movements happen, the social force model is the best-fit model to represent the crowd dynamics and the micro-mobility behavior for the evacuation of the visitors in theme parks.

\section{Simulation Study}

\section{A. Simulation environment}

The simulations of our model of theme park mobility for disaster scenarios (TP-D) are carried out to observe its characteristics. We then compare TP-D with the currently used mobility models and the 41 GPS traces (taken from the
CRAWDAD archive at Darthmouth College) collected from 11 volunteers who spent their Thanksgiving or Christmas holidays at Disney World. Each of these mobility traces have up to 10 hours of mobility data. The traces are filtered such a way that when a visitor is moving very fast, we assumed the visitor is in a vehicle traveling from one park to another. The remaining data is used for finding the set of flight lengths of each visitor where the flight length is defined as the distance between a pair of consecutive waypoints of a visitor.

The theme park mobility model (TP) [3] and self-similar least action human walk model (SLAW) [6] are used as realistic mobility models for theme parks. Random waypoint (RWP) is used as a generic model since it is the most commonly used mobility model in the network simulations.

The simulation of the model generates synthetic mobility traces of visitors in the terrain specified by the theme park map. The visitor in the theme park draws their trajectory lines while moving upon the waypoints with the goal of arriving at the gates. The dimension lengths of the maps vary from one park to another. For instance, the dimensions are close to $1000 \times 1200$ meters for Epcot and Magic Kingdom and approximately 650x750 meters for Islands of Adventure park of the Universal Studios. We used the theme park model of the Magic Kingdom. We employ the circular specification of the SFM with the angular dependency using the same parameter values proposed in [4]. Fig. 3 shows a snapshot from the simulation of 2000 visitors. Table I summarizes the simulation parameters and the parameters used for the SFM in the experiments.

TABLE I

SIMULATION PARAMETERS

\begin{tabular}{|l|l|}
\hline simulation time & $1000 \mathrm{~s}$ \\
\hline sampling time & $0.5 \mathrm{~s}$ \\
\hline number of visitors & 1000 \\
\hline min speed & $0.5 \mathrm{~m} / \mathrm{s}$ \\
\hline max speed & $2.5 \mathrm{~m} / \mathrm{s}$ \\
\hline number of red-zones & 20 \\
\hline red-zone active time & $500 \mathrm{~s}$ \\
\hline red-zone radius & $50 \mathrm{~m}$ \\
\hline random move distance & $10 \mathrm{~m}$ \\
\hline visibility & $50 \mathrm{~m}$ \\
\hline SFM - interaction strength $(\mathrm{A})$ & $0.11 \pm 0.06$ \\
\hline SFM - interaction range $(B)$ & $0.84 \pm 0.63$ \\
\hline SFM - relaxation time $(\tau)$ & $0.5 \mathrm{~s}$ \\
\hline SFM - $\lambda$ & 0.1 \\
\hline
\end{tabular}

\section{B. Simulation results}

1) Experiment 1: Flight lengths. A flight length is the distance between a pair of consecutive pause points of a visitor in its trajectory. Flight length distribution is one of the most commonly used metrics for human mobility models since the lengths of the movement of people have significant effects on the performance of the mobile networks. The total number of flights are normalized to 1000 in this simulation study for comparison among the models.

We first simulate flight length distributions of the TP-D model. We observed that the results of the simulation of the model for different simulation runs is very close, which shows the overall consistency of the simulation. Fig. 4 shows this consistency for the flight length distributions among the 5 


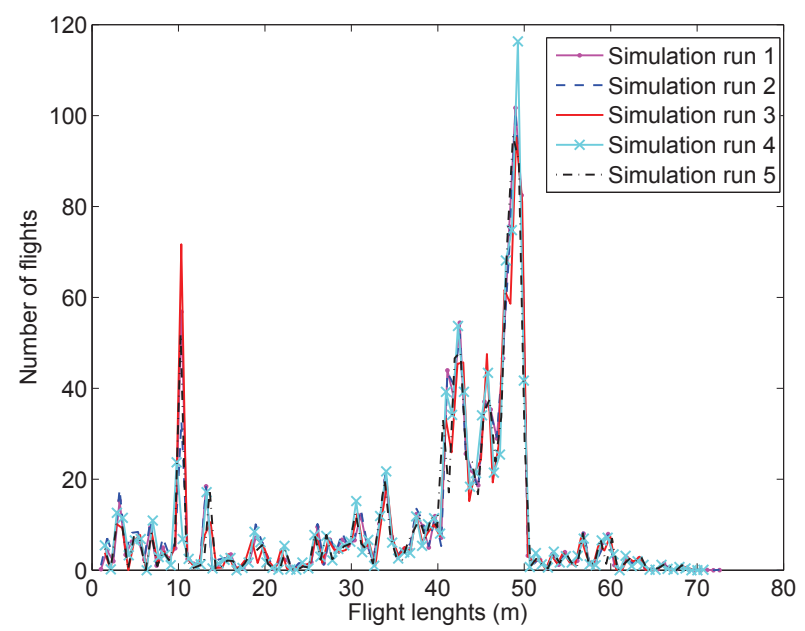

Fig. 4. Flight length distributions of the TP-D model for 5 simulation runs.

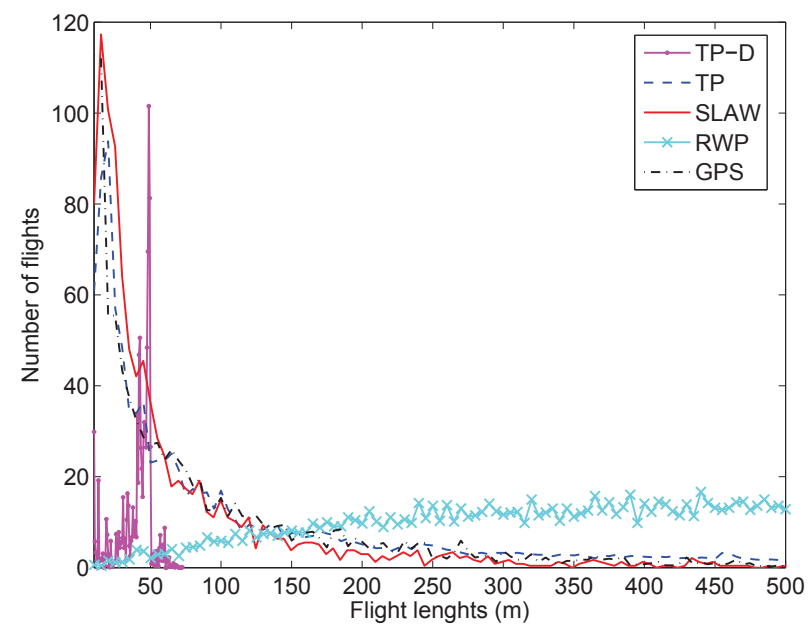

Fig. 5. Flight length distributions for TP-D, TP, SLAW, RWP, and the GPS traces.

simulation runs which are randomly selected from a set of simulation runs having the same parameter settings. The two peak points in this figure correspond to two parameters of the mobility model. The first one is the visibility parameter ( 50 meters), which can be seen in the figure as the highest peak point. This result is due to the fact that in the model, the visitors tend to select the waypoint which is closest to the target location (exit gate) among all currently visible waypoints. Therefore, the visitors mostly select the furthest waypoint from their current position in their visible area. The second highest peak point is caused by the random move distance parameter of 10 meters. The decision of selecting the random point to move is caused by red-zones and other physical obstacles.

Next, we compare the results for the simulation of our TP-D model with the synthetic traces of the other mobility simulations and the GPS traces. Fig. 5 shows that the characteristic distribution of the flight lengths does not hold in the model for disaster scenario. This is an expected outcome of the model and the peak point in TP-D is again caused by the visibility

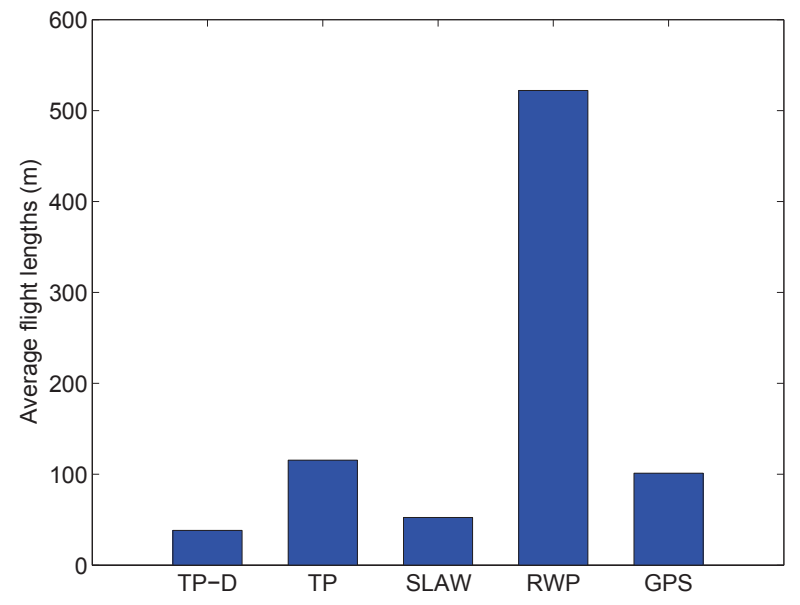

Fig. 6. Average flight lengths for TP-D, TP, SLAW, RWP, and the GPS traces.

constraint, similar to the previous figure. Overall, the flight lengths of TP-D is shorter and the range is narrower. The main reason of this difference of TP-D from the other models is the assumption of the local knowledge of the visitors. The TP-D model assumes that long flights without pausing on the way is not possible for the disaster scenario.

Fig. 6 shows that on average flight length values are still close to the results of TP, SLAW, and the GPS traces compared to the results of the RWP model. This graph shows that for GPS traces, the average flight length is close to $100 \mathrm{~m}$. In RWP, the free movement in the area causes unrealistic flights with average length of $500 \mathrm{~m}$. On the other hand, the movement of visitors is constrained with the visibility, the red-zones, and the crowd itself in TP-D, which causes the shortest flights on average.

2) Experiment 2: Evacuation times. Evacuation time is the time it takes for the visitors to reach the target (exit) points from the beginning of the simulation. The results of the evacuation times are analyzed for various values of the visibility and the number of red-zones parameters. The simulations of TP, SLAW and RWP are not used for analysis, since these models do not consider the evacuation of the visitors.

In order to see the effects of the local knowledge, we compare the TP-D model with various visibility values. We see in Fig. 7 that the increase in the knowledge causes an overall decrease in evacuation time as expected. However, after visibility value of 40 meters, this effect loses its significance.

In Fig. 8, we see that the increase in the number of redzones has a direct relation with the evacuation time. The higher numbers of red-zones constantly produce the higher evacuation times, which is an expected negative effect. The reason behind this negative effect can be easily observed by looking at a snapshot of the visitor flows in Fig. 3. Among the 7 currently active red-zones which are randomly positioned, 2 of them are located in a way that they prevent the regular flow of the visitors. This impact of preventing the visitors from moving along the road and tunneling them to other ways is the reason for the increase in the average evacuation times. 


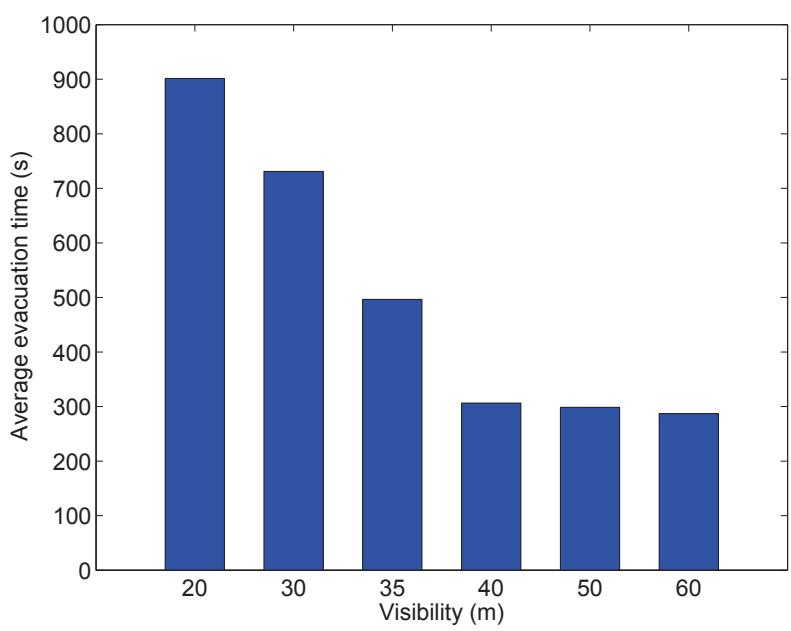

Fig. 7. Effects of the visibility on evacuation times for TP-D model.

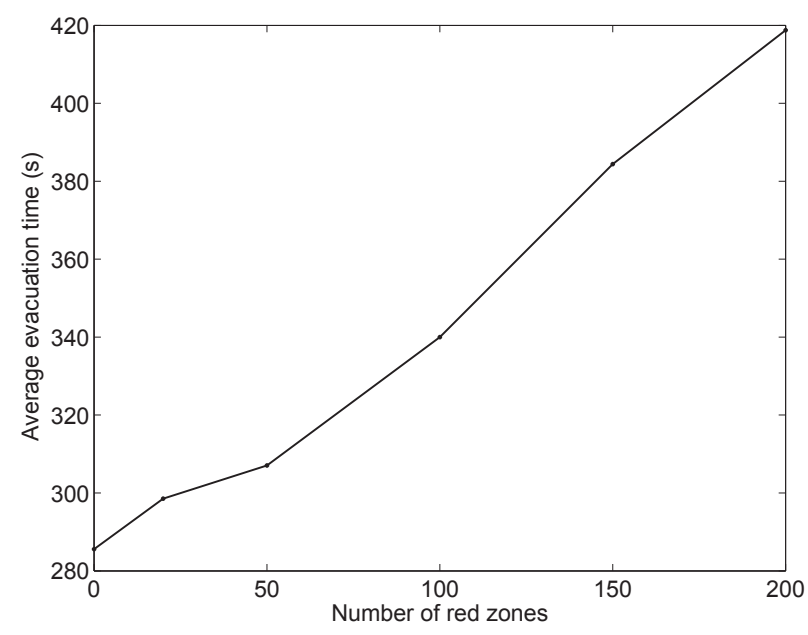

Fig. 8. Effects of the number of red-zones on evacuation times for TP-D.

\section{RELATED WORK}

In this section, we summarize the related studies for the mobility models and disaster management.

\section{A. Mobility models}

Munjal et al. [7] review the changing trends of mobility models which are recently proposed and used for simulations of opportunistic communication networks. The SLAW model is proposed by Lee et al. [6] to model the fundamental statistical features of human mobility, such as heavy-tail flight and pause-time distributions and truncated power-law intercontact times. The simulation of SLAW mobility model can be used to generate synthetic human mobility traces. This model has similar characteristics of least-action and self-similarity. Munjal et al. [8] propose a simple mobility model, SMOOTH, that represents the similar characteristics of SLAW model. Vukadinovic et al. [9] propose a simple framework to simulate mobility of theme park visitors. They use OSM for generation of maps and calibrate the framework parameters according to the GPS traces.

\section{B. Disaster management}

There exist various studies related to impacts of disaster management. Winter et al. [10] study the evacuation problem in disaster areas and propose the use of a mobile service $\mathrm{GOH}$ running on mobile phones. Benefits of communication among people are observed for the evacuation scenarios in which individuals have only the local knowledge of the environment. Patrix et al. [11] model mobility of agents and disaster area for crowd behavior detection. In this study, they model obstacles, dangers, and shelters as separate zones in their simulation of the disaster scenario. They separate the disaster area in various sub-areas such as incident site, casualties treatment area, transport zone, and hospital zone. Bagrow et al. [12] study collective response behavior and changes in communications of people in extreme emergency conditions such as bombing, plane crash, earthquake and power blackout. Patterson et al. [13] highlight models which consider effects of communities on preparedness, response, and recovery of people from disasters.

\section{CONCLUSION}

In this paper, we proposed a new mobility model (TPD) of the theme park visitors in disaster scenarios. We used real theme park maps to model the disaster environment. The mobility visitors are modeled using the theme park models and the social force model. Through extensive simulations, our model was evaluated and compared with the results of TP, SLAW, RWP, and the GPS traces.

\section{REFERENCES}

[1] G. Solmaz and D. Turgut, "Event coverage in theme parks using wireless sensor networks with mobile sinks," in Proc. of the IEEE ICC'13, June 2013, pp. 115-119.

[2] V. Vukadinovic and S. Mangold, "Opportunistic wireless communication in theme parks: a study of visitors mobility," in Proc. of the CHANTS'11, September 2011, pp. 3-8.

[3] G. Solmaz, M. Akbas, and D. Turgut, "Modeling visitor movement in theme parks," in Proc. of the IEEE LCN'12, October 2012, pp. 36-45.

[4] D. Helbing and A. Johansson, "Pedestrian, crowd and evacuation dynamics," Encyclopedia of Complexity and Systems Science, vol. 16, no. 4, pp. 6476-6495, 2010.

[5] M. Haklay and P. Weber, "OpenStreetMap: User-generated street maps." Pervasive Computing, vol. 7, no. 4, pp. 12-18, December 2008.

[6] K. Lee, S. Hong, S. J. Kim, I. Rhee, and S. Chong, "SLAW: self-similar least-action human walk," IEEE/ACM Transactions on Networking, vol. 20, no. 2, pp. 515-529, April 2012.

[7] A. Munjal, T. Camp, and N. Aschenbruck, "Changing trends in modeling mobility: a simple way to model human walks," Journal of Electrical and Computer Engineering, vol. 2012, pp. 1-16, October 2012.

[8] A. Munjal, T. Camp, and W. C. Navidi, "SMOOTH: a simple way to model human walks," ACM SIGMOBILE Mobile Computing and Communications Review, vol. 14, no. 4, pp. 34-36, November 2010.

[9] V. Vukadinovic, F. Dreier, and S. Mangold, "A simple framework to simulate the mobility and activity of theme park visitors," in Proc. of the WSC'11, December 2011, pp. 3248-3260.

[10] S. Winter, K.-F. Richter, M. Shi, and H.-S. Gan, "Get me out of here: collaborative evacuation based on local knowledge," in Proc. of the IWCTS'11, November 2011, pp. 35-42.

[11] J. Patrix, A.-I. Mouaddib, and S. Gatepaille, "Detection of primitive collective behaviours in a crowd panic simulation based on multi-agent approach." International Journal of Swarm Intelligence Research, vol. 3, no. 3, pp. 50-65, June 2012.

[12] A.-L. B. James P. Bagrow, Dashun Wang, "Collective response of human populations to large-scale emergencies," PLOS ONE, vol. 6, no. 3, March 2011.

[13] O. Patterson, F. Weil, and K. Patel, "The role of community in disaster response: Conceptual models," Population Research and Policy Review, vol. 29, no. 2, pp. 127-141, April 2010. 